\title{
First Results of Optical Monitoring of 3C 390.3
}

\author{
M. Dietrich
}

Landessternwarte Heidelberg, Königstuhl, D-69117 Heidelberg, Germany

P.T. O'Brien

Department of Physics \& Astronomy, University of Leicester, UK

K. M. Leighly

Institute of Physical and Chemical Research (RIKEN), Japan

\section{Introduction}

In late autumn 1994, the International AGN Watch started a multiwavelength monitoring campaign on $3 \mathrm{C} 390.3$, the first radio-loud AGN to be selected for high temporal-resolution monitoring from the radio domain to $\mathrm{X}$-ray energies. This AGN is is of particular interest since significant variations have been observed on both short and long time scales in both the line fluxes and line profiles (Barr et al. 1980, Veilleux \& Zheng 1991).

\section{Observations and Analysis}

In the optical, spectroscopic data and broad-band flux measurements in $B, V, R$, and $I$ were obtained. The brightness of $3 \mathrm{C} 390.3$ was calculated relative to the stars in the field. The $R$ magnitudes are based on observations recorded at Calar Alto, Spain, in 1994 December. Generally, AGN spectra are intercalibrated using narrow forbidden emission lines since these lines can be taken as constant on time scales of decades (Peterson 1993). Since the $[\mathrm{O} \mathrm{III]} \lambda \lambda 4959,5007 \mathrm{flux}$ is variable on time scales of several months (Zheng et al. 1995), these lines were studied in detail. We found that they were constant within $\sim 2 \%$ during 1995 (Dietrich et al. 1996). The spectra were therefore intercalibrated by using the narrow forbidden emission lines. 3C 390.3 was also observed with IUE for 14 months (O'Brien et al. 1996) and with ROSAT for nine months in 1995 (Leighly et al. 1996). Radio data were also obtained during a 3-month MERLIN campaign.

The photometric light curves in the $B, V, R$, and $I$ bands show significant variations. The brightness increased nearly monotonically (see Fig. 1). Variations on time scales of weeks were superimposed on these general trend. The parameter $F_{\text {var }}$ indicates that the variability amplitude decreased with wavelength; $F_{\mathrm{var}}(V)=0.158, F_{\mathrm{var}}(R)=0.102, F_{\mathrm{var}}(I)=0.087$. Light curves of the broad Balmer emission lines, He Ir $\lambda 4686$, and He I $\lambda 5876$ (Dietrich et al. 1996) were also measured. The $\mathrm{H} \beta$ flux variations are presented in Fig. 1 as an example. The general variability pattern of the other emission lines is nearly identical, but the scatter is larger due to the weaker line strength. 


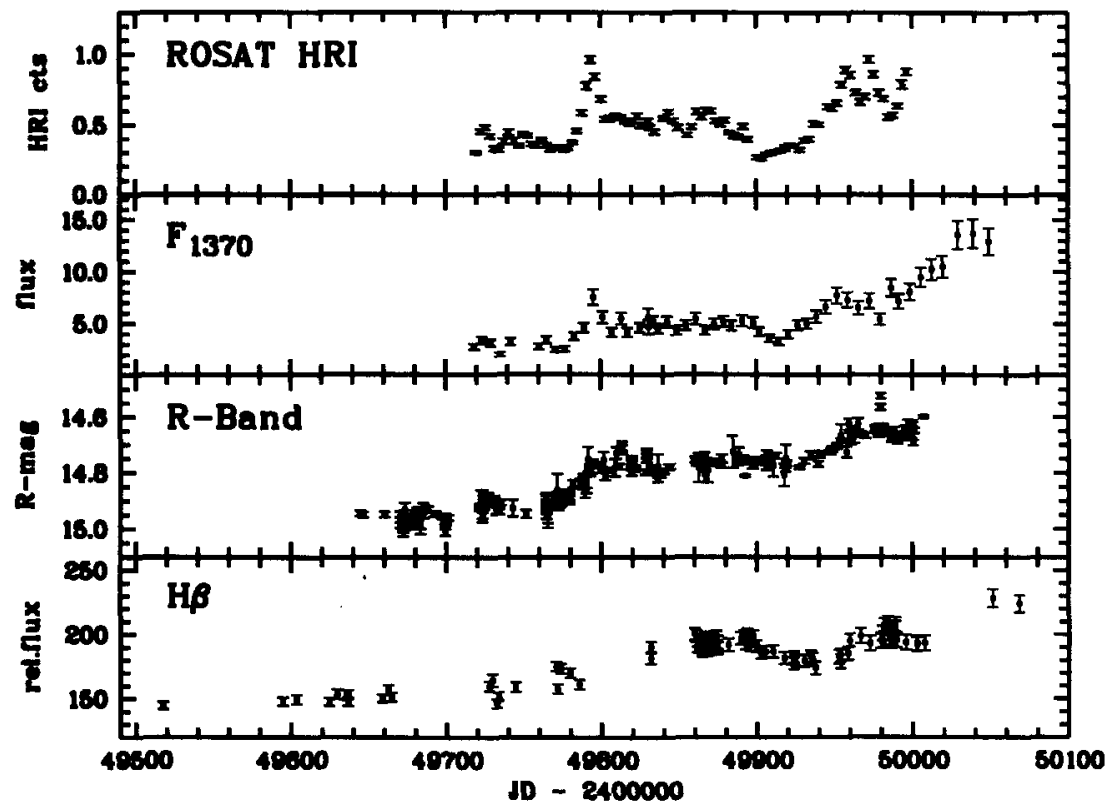

Figure 1. Light curves of soft $\mathrm{X}$-ray, $1370 \AA, R$ band, and $\mathrm{H} \beta$ flux variations.

As a first step in analyzing the variations of 3C 390.3, cross-correlation functions (CCFs) have been calculated. The X-ray flux (Leighly et al. 1996) was assumed to represent the continuum that drives the variations of the broad emission-line flux. Cross-correlation of the X-ray and ultraviolet (1370 $\AA$ ) continua (O'Brien et al. 1996) yields a delay of $\sim 0.4$ days. The variations of the integrated $\mathrm{H} \beta$ flux were delayed by 36 days with respect to the X-ray flux, based on the centroid of the corresponding CCF.

Acknowledgments. This work has been supported by DFG grant SFB 328.

\section{References}

Barr, P., et al. 1980, MNRAS, 193, 549.

Dietrich, M., et al. 1996, in preparation.

Leighly, K.M., et al. 1996, in preparation.

O'Brien, P.T., et al. 1996, in preparation.

Veilleux, S., \& Zheng, W. 1991, ApJ, 377, 89.

Peterson, B.M. 1993, PASP, 105, 247.

Zheng, W., Pérez, E., Grandi, S.A., \& Penston, M.V. 1995, AJ, 109, 2355. 